\title{
UMIDADE CRÍTICA DE COMPACTAÇÃO E DENSIDADE DO SOLO MÁXIMA EM RESPOSTA A SISTEMAS DE MANEJ O NUM LATOSSOLO ROXO(1)
}

\author{
L. H. A. FIGUEIREDO(2), M. S. DIAS J UNIOR ${ }^{(3)}$ \& M. M. FERREIRA(4)
}

\begin{abstract}
RESUMO
O termo compactação do solo refere-se à compressão do solo não saturado durante a qual existe um aumento da densidade do solo em conseqüência da redução de seu volume, pela expulsão do ar causado pelo manejo inadequado. Com o objetivo de estudar o efeito de diferentes sistemas de manejo na densidade do solo máxima ( $\left.D s_{\text {máx }}\right)$, e na umidade crítica de compactação $\left(U_{\text {crít }}\right)$, realizou-se um experimento em um Latossolo Roxo da Região de Lavras (MG), submetido aos seguintes sistemas de manejo: preparo com arado de discos (AD), preparo com escarificador (CM), semeadura direta (SD), preparo com arado de aivecas (AA) e preparo intensivo com grade (GD). 0 experimento foi instalado, em novembro de 1994, em blocos casualizados. Foram realizadas três amostragens em 1996: em janeiro, antes da semeadura do feijão; em abril, antes da colheita do feijão, e em novembro, antes da semeadura do milho. As amostras deformadas foram coletadas nas camadas de 0-0,07 e 0,20-0,27 $\mathrm{m}$ de profundidade nos sistemas de manejo AD, CM e AA. No sistema de manejo SD, as amostras deformadas foram coletadas nas camadas de 0-0,07 e 0,25-0,32 m de profundidade e, no caso do sistema GD, nas camadas de 0-0,07 e 0,15-0,22 m de profundidade. A partir das curvas de compactação, obteve-se a densidade do solo máxima. Determi naramse, ainda, o teor de argi la e matéria orgânica (MO), o limite de plasticidade (LP) e a água retida a - 0,033e-0,01 MPa. Os valores de ( $\left.D s_{\text {máx }}\right)$, $\left(U_{\text {crít }}\right)$ para os diferentes sistemas de manejo, época de coleta e profundidades, não foram estatisticamente diferentes, não sendo, portanto, influenciados pelos teores de argila e M.O. Verificou-se que a $U_{\text {crít }}$ correspondea $90 \%$ do LP e a $90 \%$ da água retida a - 0,01 MPa é aproxi madamente igual à água retida a - 0,033 $\mathrm{MPa}$.
\end{abstract}

Termos de Indexação: preparo do solo, ensaio de proctor normal, propriedades físicas.

\footnotetext{
(1) Parte da Tese de Mestrado apresentada pelo primeiro autor ao DCS/UFLA, com o auxilio da CAPES. Recebido para a publicação em maio de 1998 e aprovado em maio de 2000.

(2) Engenheiro-Agrônomo, Mestrado no CPGNSP, DCS/Universidade Federal de Lavras - UFLA. Caixa Postal 37, CEP 37200-000 Lavras (MG).

(3) Professor Adjunto IV do DCS/UFLA. Bolsista do CNPq.

(4) Professor Titular do DCS/UFLA. Bolsista do CNPq.
} 


\title{
SUMMARY: CRITICAL MOISTURE CONTENT AND MAXIMUM DRY BULK DENSITY IN RESPONSE TO SOIL MANAGEMENT SYSTEMS IN A DUSKY RED LATOSOL
}

\begin{abstract}
Theterm soil compaction refers to thecompression of an unsaturated soil during which an increase of the bulk density of soil occurs in consequence of the reduction of its volume, due to the expulsion of the air caused by inadequate management. To study the effect of different soil management systems on maximum dry bulk density and optimum moisture content, an experiment was conducted in a Dusky Red Latosol of the Lavras (MG, Brazil) region, submitted to the fol lowing management systems: pl ow disk (AD), plow moldboard $(A A)$, minimum tillage (CM), no tillage (SD) and harrow disk (GD). The experiment was installed in N ovember, 1994. Disturbed soil samples weretaken in 1996: in J anuary before bean plantation; in April, before bean harvest; and in N ovember, beforecorn planting. Soil samples were taken from the 0 to 0.07 layer and from the 0.20 to $0.27 \mathrm{~m}$ layer, under management systems AD, CM and AA. For management system SD, thedisturbed soil samples weretaken from thelayer of 0-0.07 and 0.25-0.32 m depth and for GD from thelayer of 0 to 0.07 and 0.15 to $0.22 \mathrm{~m}$ depth. Maximum dry bulk density ( $\left(\mathrm{s}_{\text {máx }}\right)$ and critical moisture content $\left(\mathrm{U}_{\text {crit }}\right)$ were obtained from the soil compaction curves. Clay content, organic matter, plasticity limit (PL) and water content retained at - 0.033 and - $0.01 \mathrm{MPa}$ were also determined. The Ds $s_{\text {máx }}, U_{\text {crít }}$ for the soil management systems, time of sampling and depth were not statistically different, showing that they were not influenced by clay and organic matter content. $U_{\text {crít }}$ was observed to occur at $90 \%$ of $\mathrm{PL}$ and at $90 \%$ of the water retained at - $0.01 \mathrm{MPa}$, being approximately equal to the water content retained at - $0.033 \mathrm{MPa}$.
\end{abstract}

Index terms: soil tillagesystems, standard Proctor test, physical properties.

\section{INTRODUÇÃO}

Nas últimas décadas, com a expansão da fronteira agrícola e com a exploração de duas ou três safras anuais, observou-se uma crescente utilização de máquinas agrícolas, desde o preparo do solo até à col heita, além de um aumento nos pesos e potências dos tratores (Mantovani, 1987). I sso contribuiu para aumentar as áreas com problemas de compactação, provavel mente pela ausência de um cronograma de trabalho bem definido ou de modelos capazes de estimar a capacidade de suporte de carga do solo.

Por essa razão, muitas vezes, o preparo do solo é realizado sem levar em conta sua umidade, a qual é um fator controlador da compactação (Dias J unior \& Pierce, 1996). Para evitá-la, o preparo do solo deve ser efetuado em condições de friabilidade (Resende,1997), na qual este apresenta baixa resistência ao preparo ealta a moderada capacidade de suporte de carga e resistência à compressão (Larson et al., 1994).

No estudo da compactação do solo, vários ensaios de laboratório têm sido usados. Um dos mais utilizados é o Proctor normal (Dias J unior, 1996). Neste ensaio, para uma mesma energia de compactação, a densidade do solo depende de sua umi dade no momento da compactação (Vargas,1977). Plotando os valores de umidade versus densidade do solo, obtém-se a curva de compactação, da qual são obtidas a umidade crítica de compactação e a densidade do sol o máxima correspondente.

A curva de compactação depende de vários fatores, tais como: energia de compactação, textura ematéria orgânica do sol o (Silva et al., 1986; Ohu et al., 1986; Ekwue \& Stone, 1997).

A energia de compactação utilizada no ensaio de Proctor normal é equivalente a um rolo tipo "pé de carneiro" leve ( 5 a $7 \mathrm{t}$ ), passando cerca de 12 vezes sobre uma camada de sol o com 0,30 m de espessura (Vargas, 1977). J á na agricultura, a energia de compactação representa, aproximadamente, 15 passadas de um trator com 0,727 t na roda (pressão de $140 \mathrm{kPa}$ ) em solo franco-arenoso (Raghavan et al., 1976).

As curvas de compactação do solo assemel hamse quanto à forma, independentemente da classe de solo (Caputo, 1973). Segundo Pacheco \& Dias J unior (1990), à medida que se adicionava areia a um Latossol o Vermelho-Amarelo, os valores da densi dade do sol o máxima aumentavam e os da umi dade crítica de compactação dimi nuíam. De acordo com Silva et al. (1986) e Ekwue \& Stone (1997), maiores teores de argila e de matéria orgânica do solo provocam redução na amplitude das curvas de compactação, diminuindo, conseqüentemente, a densidade do solo máxima e aumentando a umidade crítica de 
compactação. Isto ocorre pelo fato de a argila e a matéria orgânica influenciarem o poder deadsorção de água do solo (Silva et al., 1986). Segundo Ekwue \& Stone (1995) e Stone \& Ekwue (1993), a redução da densidade do solo máxima e o aumento da umidade crítica de compactação com o aumento da matéria orgânica são atribuídos ao seu baixo valor de densidade e ao aumento da umidade do solo promovido pela sua adição.

A literatura brasileira sobre o uso do ensaio de Proctor normal para fins agrícolas éescassa. Assim, este trabalho teve como objetivos: estudar o efeito de diferentes sistemas de manejo do sol ona umidade crítica de compactação e na densidade do solo máxima de um Latossolo Roxo.

\section{MATERIAL E MÉTODOS}

Este estudo foi realizado num Latossolo Roxo (LR), originário de gabro (Rocha, 1982), localizado no Campus da UniversidadeF ederal de Lavras, MG $\left(21^{\circ} 13^{\prime} \mathrm{S}\right.$ e $\left.44^{\circ} 57^{\prime} \mathrm{W}\right)$, com al titude média em torno de 918 metros, clima Cwb, segundo classificação de Köppen (Castro Neto, 1982).

Antes da instalação do experimento, a área era usada há mais de quinze anos, sendo o milho a principal cultura. O preparo dosolo era feito por meio de uma gradagem, seguida de uma aração com arado dedisco com 0,70 m de diâmetro e, a seguir, aplicavaseuma grade de 14 discos de 26 " de bordos recortados, atuando a 0,15 $\mathrm{m}$ de profundidade, tantas vezes quantas fossem necessárias para destorroamento e nivelamento do terreno. Antes da instalação do experimento, a área foi subsolada a uma profundidade aproximada de 0,40 m, submetida a uma calagem, conforme recomendação da Comissão de Fertilidade do Sol o do Estado de Minas Gerais (1989), e deixada em repouso por dois meses. A área experimental foi de 1,08 ha e cada parcela ocupou $720 \mathrm{~m}^{2}$. As parcelas foram separadas entre si por carreador de $20 \mathrm{~m}$ de largura e os blocos, por terraços. Os tratamentos constituíram-se dos sistemas de manejo:

Semeadura direta (SD) - o solo foi revolvido apenas aolongo das linhas, por ocasião da semeadura. Utilizou-se uma semeadora-adubadora marca J umil, model o 2040, equipada com acessórios para semeadura direta.

Preparo conservacionista com escarificador (CM) - utilizou-se, neste sistema, o escarificador de hastes rígi das, marca Maschietto, model oSDM-7-A, de arrasto, com cinco hastes, a uma profundidade de $20 \mathrm{~cm}$.

Preparo convencional com arado de aivecas (AA) - utilizou-se, no preparo primário do solo, um arado de três aivecas, fixo, marca Maschietto, modelo A-3, a uma profundidade de $20 \mathrm{~cm}$.
Preparo convencional com arado de disco (AD) - utilizou-se, no preparo primário do solo, o arado com três discos de $70 \mathrm{~cm}$ de diâmetro, reversível, marca Santa I sabel, modelo 328, a uma profundidade de $20 \mathrm{~cm}$.

Preparo Intensivo com grades intermediárias (GD) - utilizou-se, no preparo primário do solo, uma grade intermediária, marca Marchesan, modelo GAP, de arrasto, a uma profundidade de $15 \mathrm{~cm}$.

Semeou-se milho tardio em janeiro de 1995, em novembro de 1995, o solo foi novamente preparado, tendo sido os tratamentos repetidos em novembro de 95 e 96, plantando-se feijão, em janeiro de 96, e milho, em dezembro. A seqüência das culturas foi milho tardio, feijão e milho.

Foram realizadas três amostragens: uma, em janeiro de 1996, antes da semeadura do feijão; em abril, antes da colheita de feijão e, em novembro, antes da semeadura do milho. Coletaram-se amostras deformadas, nas camadas de 0-0,07 e 0,200,27 m de profundidade, para os sistemas de manejo AD, CM e AA. Para o SD, nas camadas de 0-0,07 e $0,25-0,32 \mathrm{~m}$ e, para o caso do GD, nas camadas de $0-0,07$ e $0,15-0,22 \mathrm{~m}$.

Os valores da densidade do solo em relação à umidade obtidos por meio do ensaio de Proctor normal com reuso do solo (Stancati et al., 1981) foram ajustados a uma equação do segundo grau, obtendose a curva de compactação e, a partir desta, determinaram-se a umi dade crítica de compactação e a densidade do sol o máxima correspondente.

Foram determinados também a textura pelo método da pipeta (Day, 1965) (Quadro 1), matéria orgânica (Raij \& Quaggio, 1983), água retida a -0,010 e -0,033 MPa (Klute, 1986) e limite de plasticidade (Sowers, 1965).

Usou-se o delineamento experimental de blocos ao acaso, com cinco tratamentos aplicados em faixa e três repetições. A análise estatística seguiu o modelo:

$$
\begin{aligned}
& y_{i j k l}=m+b_{i}+a_{j}+E a_{i j}+p_{k}+E b_{i k}+ \\
& +c_{l}+E c_{i l}+(a p)_{j k}+E d_{i j k}+(a c)_{j l}+ \\
& +E e_{i j l}+(p c)_{k l}+E f_{i k l}+(a p c)_{j k l}+E g_{i j k l}
\end{aligned}
$$

em que:

$\mathrm{y}_{\mathrm{ijkl}}$ é a observação referente ao bloco i, preparo j, profundidade $k$, col eta l;

m é a média geral do experimento (efeito fixo);

$b_{i}$ é o efeito do bloco $i$;

a é o preparo; efeito fixo, com cinco níveis de preparo; $p$ é a profundidade; efeito fixo; com dois níveis de profundidade;

c é a coleta; efeito fixo, com três épocas de coleta;

Ea é o erro referente ao fator preparo; nid $\sim \mathrm{n}\left(0, \sigma \mathrm{a}^{2}\right)$;

$E b$ é o erro referente ao fator profundidade; nid $\sim n$ $\left(0, \sigma b^{2}\right)$; 
Ec é o erro referente ao fator col eta; nid $\sim n\left(0, \sigma c^{2}\right)$; $\mathrm{Ed}$ é o erro referente à interação preparo e profundidade; nid $\sim \mathrm{n}\left(0, \sigma \mathrm{d}^{2}\right)$;

Ee é o erro referente à interação preparo e coleta, nid $\sim \mathrm{n}\left(0, \sigma \mathrm{e}^{2}\right)$;

Ef é o erro referente à interação profundidade e col eta ; nid n $\left(0, \sigma \mathrm{f}^{2}\right)$;

Eg é o erro referente à interação preparo, profundidade e col eta; nid $\sim \mathrm{n}\left(0, \sigma g^{2}\right)$.

Utilizou-se o método da máxima verossimilhação restrita (REML), em razão do tipo de del ineamento utilizado neste estudo (Latour et al., 1994). Cada combinação de níveis de fatores foi estimada por um intervalo de confiança (IC), seguindo a distribuição t de student, a 95\%. As comparações basearam-se na sobreposição dos ICs. Os procedimentos de cálculos foram realizados pela Proc Mixed do SAS (SAS, 1990).

\section{RESULTADOS E DISCUSSÃO}

Para um mesmo sistema de manejo, os teores de argila, silte e areia não diferiram nas duas profundidades estudadas (Quadro 1).

A partir das curvas de compactação, a umidade crítica de compactação ea densidade do sol o máxima foram obtidas (Quadro 2). Esses valores, para os diferentes sistemas de manejo, época de coleta e profundidades, não foram estatisticamente

Quadro 1. Teores de argila, silte e areia de um Latossolo Roxo, considerando o sistema de manejo e a profundidade

\begin{tabular}{|c|c|c|c|c|}
\hline $\begin{array}{c}\text { Sistema } \\
\text { de manejo }\end{array}$ & Profundidade & Argila & Silte & Areia \\
\hline & $\mathrm{m}$ & \multicolumn{3}{|c|}{$\mathrm{g} \mathrm{kg}^{-1}$} \\
\hline$A D^{(1)}$ & $\begin{array}{r}0-0,07 \\
0,20-0,27\end{array}$ & $\begin{array}{l}590 a^{(2)} \\
660 a\end{array}$ & $\begin{array}{l}220 a \\
150 a\end{array}$ & $\begin{array}{l}190 a \\
190 a\end{array}$ \\
\hline CM & $\begin{array}{r}0-0,07 \\
0,20-0,27\end{array}$ & $\begin{array}{l}620 a \\
710 a\end{array}$ & $\begin{array}{l}180 a \\
130 a\end{array}$ & $\begin{array}{l}120 a \\
160 a\end{array}$ \\
\hline SD & $\begin{array}{r}0-0,07 \\
0,25-0,32\end{array}$ & $\begin{array}{l}650 a \\
670 a\end{array}$ & $\begin{array}{l}160 a \\
150 a\end{array}$ & $\begin{array}{l}190 a \\
180 a\end{array}$ \\
\hline AA & $\begin{array}{r}0-0,07 \\
0,20-0,27\end{array}$ & $\begin{array}{l}690 a \\
700 a\end{array}$ & $\begin{array}{l}130 a \\
120 a\end{array}$ & $\begin{array}{l}180 a \\
180 a\end{array}$ \\
\hline GD & $\begin{array}{r}0-0,07 \\
0,15-0,22\end{array}$ & $\begin{array}{l}630 a \\
700 a\end{array}$ & $\begin{array}{l}180 a \\
130 a\end{array}$ & $\begin{array}{l}190 a \\
170 a\end{array}$ \\
\hline
\end{tabular}

diferentes (Quadro 2), pel o fato de os teores deargila e de matéria orgânica entre as épocas decol etas das amostras (Quadros 1 e 2) não terem sido estatisticamente diferentes entre si. Assim, essas características não influenciaram a densidade do solo máxima e a umidade crítica de compactação. Apesar disso, no caso da matéria orgânica, houve interação dos sistemas de manejo e a profundidade amostrada, sendo os teores médios maiores na camada superficial dos preparos conservacionista com escarificador e semeadura direta (Quadro 2).

A umidade crítica de compactação (Quadro 2) é menor do que o limite de plasticidade (Quadro 3), fato também observado por Gamero (1982), Stone \& Ekwue (1993) e Ekwue \& Stone (1997). Sendo o limite de plasticidade o limite superior da zona de friabilidade do solo, verifica-se que a umidade crítica de compactação está contida na faixa de umidade onde o tráfego de máquinas érealizado. Assim, podese sugerir que o tráfego de máquinas não seja realizado quando a umidade do solo for aproximadamente igual ao limite de plasticidade, o que evitaria maiores riscos de compactação do solo.

Pelo fato de o ensaio de Proctor normal não ser uma análise de rotina em laboratórios de física do solo, pesquisadores têm buscado maneiras alternativas para determinar a umidade crítica de compactação. Assim, têm-se utilizado alguns atributos do solo, tais como: $90 \%$ do limite de plasticidade, $90 \%$ da água retida a - 0,01 MPa ou a água retida a - 0,033 MPa na tentativa de obtenção da umidade crítica de compactação. Segundo Ojeniyi \& Dexter (1979), a umidade do solo que prejudica os trabalhos com máquinas está próxima a $90 \%$ do limite de plasticidade. Observando os valores da umi dade crítica de compactação (Quadro 2), verificase que estes estão próximos dos valores de umidade igual a $90 \%$ do limite de plasticidade (Quadro 3). Com base nestes resultados, aconsel ha-se que, para o solo em estudo, as operações motomecanizadas devam ser executadas quando a umi dade for menor do que 0,29 kg kg-1, evitando, assim, sua compactação.

Outra característica física que tem si do utilizado para estimar a umidade crítica de compactação é a água retida a $-0,033$ ou $90 \%$ da água retida a $-0,010 \mathrm{MPa}$. Nos quadros 2 e 3, observa-se que a umidade crítica de compactação está próxima ao val or da água retida a - 0,033 MPa, concordando com os resultados observados por Campbell et al. (1980), Howard et al . (1981) ePereira (1994). Por outrolado, Sediyama et al. (1979) verificaram que os dias favoráveis ao tráfego de máquinas no solo são aqueles em quea umidade deste seja inferior ou igual a $90 \%$ da capacidade de campo. Considerando a capacidade de campo como a água retida a -0,01 MPa e analisando comparativamente os quadros 2 e 3 , notou-se que a umidade crítica de compactação estava próxima de $90 \%$ da água retida a -0,01 MPa. Assim, as operações motomecanizadas deveriam ser 


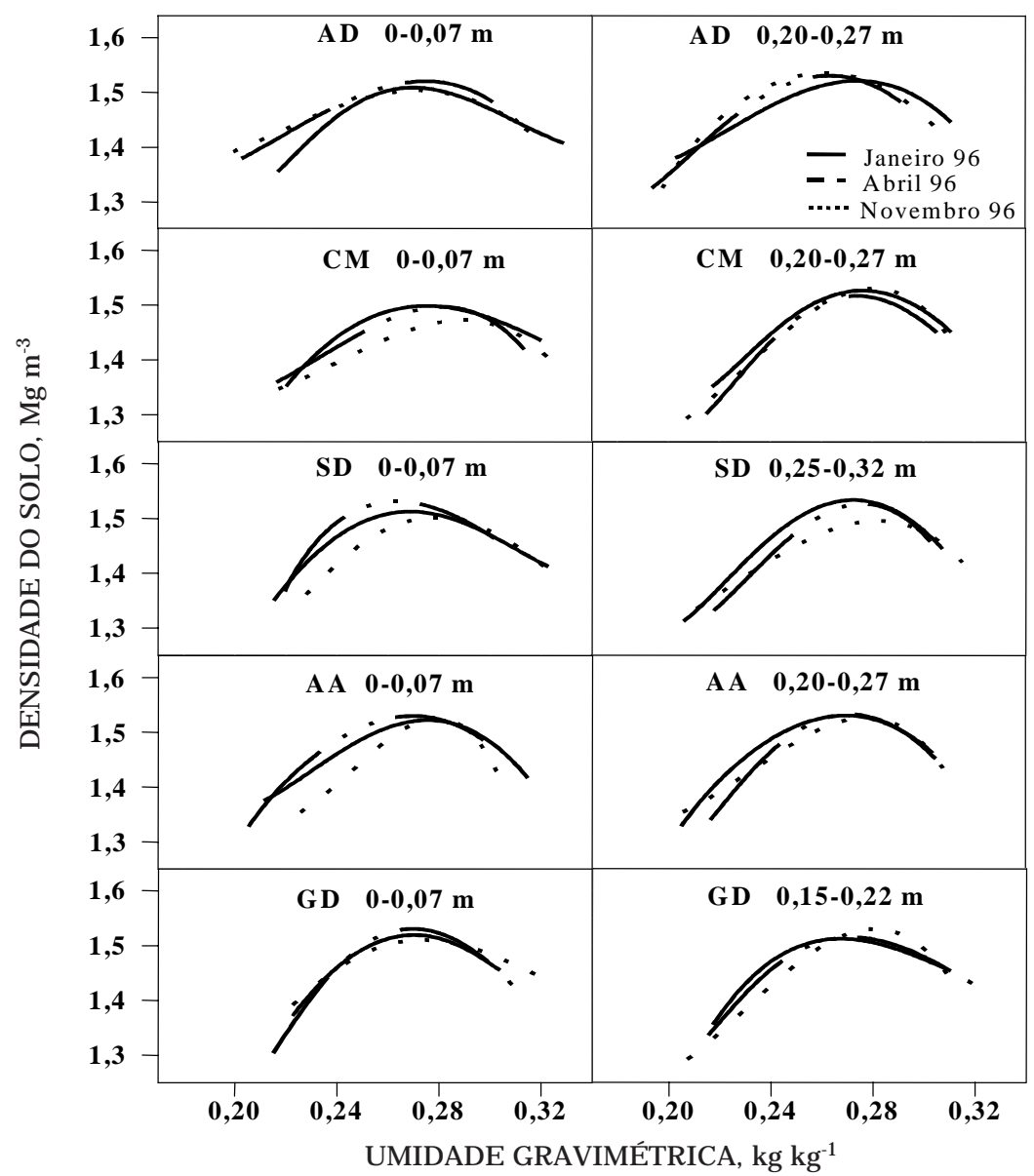

Figura 1. Curvas de compactação de um L atossolo Roxo, considerando o sistema de manejo, a profundidade ea época de amostragem (AD: preparo com arado de discos, CM: preparo com escarificador, SD: semeadura direta, AA: preparo com arado de aivecas, GD: preparo intensivo com grade).

Quadro 2. Umidade crítica de compactação $\left(U_{c r i t}\right)$, densidade do solo máxima $\left(D_{\text {máx }}\right)$ e matéria orgânica (M.O.) de um Latossolo Roxo, considerando o sistema de manejo, a profundidade ea época de amostragem

\begin{tabular}{|c|c|c|c|c|c|c|c|c|c|c|c|}
\hline \multirow{2}{*}{$\begin{array}{c}\text { Sistema } \\
\text { de manejo }\end{array}$} & \multirow{2}{*}{ Profundidade } & \multicolumn{3}{|c|}{ J aneiro/96 } & \multicolumn{3}{|c|}{ Abril/96 } & \multicolumn{3}{|c|}{ Novembro/96 } & \multirow{2}{*}{$\begin{array}{l}\text { M.O. } \\
\text { média }\end{array}$} \\
\hline & & $\mathbf{U}_{\text {crít }}$ & DSmáx & M.O. & $\mathbf{U}_{\text {crít }}$ & Dsmáx & M.o. & $\mathbf{U}_{\text {crít }}$ & Dsmáx & M.O. & \\
\hline & $\mathrm{m}$ & $\mathrm{kg} \mathrm{kg}^{-1}$ & $\mathrm{Mg} \mathrm{m}^{-3}$ & $\mathrm{~g} \mathrm{~kg}^{-1}$ & $\mathrm{~kg} \mathrm{~kg}^{-1}$ & $\mathrm{Mg} \mathrm{m}^{-3}$ & $\mathrm{~g} \mathrm{~kg}^{-1}$ & $\mathrm{~kg} \mathrm{~kg}^{-1}$ & $\mathrm{Mg} \mathrm{m}^{-3}$ & $\mathrm{~g} \mathrm{~kg}^{-1}$ & $\mathrm{~g} \mathrm{~kg}^{-1}$ \\
\hline$A D^{(1)}$ & $\begin{array}{r}0-0,07 \\
0,20-0,27\end{array}$ & $\begin{array}{l}0,27 \mathrm{Aa}^{(2)} \\
0,28 \mathrm{Aa}\end{array}$ & $\begin{array}{l}1,51 \mathrm{Aa} \\
1,52 \mathrm{Aa}\end{array}$ & $\begin{array}{l}28 \mathrm{~A} \\
26 \mathrm{~A}\end{array}$ & $\begin{array}{l}0,28 \mathrm{Aa} \\
0,27 \mathrm{Aa}\end{array}$ & $\begin{array}{l}1,52 \mathrm{Aa} \\
1,53 \mathrm{Aa}\end{array}$ & $\begin{array}{l}29 A \\
23 A\end{array}$ & $\begin{array}{l}0,28 \mathrm{Aa} \\
0,27 \mathrm{Aa}\end{array}$ & $\begin{array}{l}1,50 \mathrm{Aa} \\
1,54 \mathrm{Aa}\end{array}$ & $\begin{array}{l}29 A \\
23 A\end{array}$ & $\begin{array}{l}29 a \\
24 a\end{array}$ \\
\hline CM & $\begin{array}{r}0-0,07 \\
0,20-0,27\end{array}$ & $\begin{array}{l}0,28 \mathrm{Aa} \\
0,28 \mathrm{Aa}\end{array}$ & $\begin{array}{l}1,50 \mathrm{Aa} \\
1,53 \mathrm{Aa}\end{array}$ & $\begin{array}{l}29 A \\
24 A\end{array}$ & $\begin{array}{l}0,28 \mathrm{Aa} \\
0,27 \mathrm{Aa}\end{array}$ & $\begin{array}{l}1,50 \mathrm{Aa} \\
1,52 \mathrm{Aa}\end{array}$ & $\begin{array}{l}32 \mathrm{~A} \\
22 \mathrm{~A}\end{array}$ & $\begin{array}{l}0,29 \mathrm{Aa} \\
0,28 \mathrm{Aa}\end{array}$ & $\begin{array}{l}1,47 \mathrm{Aa} \\
1,53 \mathrm{Aa}\end{array}$ & $\begin{array}{l}31 A \\
23 A\end{array}$ & $\begin{array}{l}31 \mathrm{a} \\
23 \mathrm{a}\end{array}$ \\
\hline SD & $\begin{array}{r}0-0,07 \\
0,25-0,32\end{array}$ & $\begin{array}{l}0,27 \mathrm{Aa} \\
0,27 \mathrm{Aa}\end{array}$ & $\begin{array}{l}1,51 \mathrm{Aa} \\
1,53 \mathrm{Aa}\end{array}$ & $\begin{array}{l}30 A \\
22 A\end{array}$ & $\begin{array}{l}0,26 \mathrm{Aa} \\
0,28 \mathrm{Aa}\end{array}$ & $\begin{array}{l}1,53 \mathrm{Aa} \\
1,53 \mathrm{Aa}\end{array}$ & $\begin{array}{l}31 \mathrm{~A} \\
20 \mathrm{~A}\end{array}$ & $\begin{array}{l}0,28 \mathrm{Aa} \\
0,28 \mathrm{Aa}\end{array}$ & $\begin{array}{l}1,50 \mathrm{Aa} \\
1,50 \mathrm{Aa}\end{array}$ & $\begin{array}{l}33 A \\
28 A\end{array}$ & $\begin{array}{l}31 \mathrm{a} \\
23 \mathrm{~b}\end{array}$ \\
\hline AA & $\begin{array}{r}0-0,07 \\
0,20-0,27\end{array}$ & $\begin{array}{l}0,28 \mathrm{Aa} \\
0,27 \mathrm{Aa}\end{array}$ & $\begin{array}{l}1,52 \mathrm{Aa} \\
1,53 \mathrm{Aa}\end{array}$ & $\begin{array}{l}25 A \\
23 A\end{array}$ & $\begin{array}{l}0,27 \mathrm{Aa} \\
0,27 \mathrm{Aa}\end{array}$ & $\begin{array}{l}1,53 \mathrm{Aa} \\
1,53 \mathrm{Aa}\end{array}$ & $\begin{array}{l}25 \mathrm{~A} \\
23 \mathrm{~A}\end{array}$ & $\begin{array}{l}0,28 \mathrm{Aa} \\
0,28 \mathrm{Aa}\end{array}$ & $\begin{array}{l}1,53 \mathrm{Aa} \\
1,53 \mathrm{Aa}\end{array}$ & $\begin{array}{l}25 A \\
26 A\end{array}$ & $\begin{array}{l}25 a \\
24 a\end{array}$ \\
\hline GD & $\begin{array}{r}0-0,07 \\
0,15-0,22\end{array}$ & $\begin{array}{l}0,27 \mathrm{Aa} \\
0,27 \mathrm{Aa}\end{array}$ & $\begin{array}{l}1,52 \mathrm{Aa} \\
1,51 \mathrm{Aa}\end{array}$ & $\begin{array}{l}26 \mathrm{~A} \\
23 \mathrm{~A}\end{array}$ & $\begin{array}{l}0,27 \mathrm{Aa} \\
0,27 \mathrm{Aa}\end{array}$ & $\begin{array}{l}1,53 \mathrm{Aa} \\
1,51 \mathrm{Aa}\end{array}$ & $\begin{array}{l}30 \mathrm{~A} \\
25 \mathrm{~A}\end{array}$ & $\begin{array}{l}0,27 \mathrm{Aa} \\
0,28 \mathrm{Aa}\end{array}$ & $\begin{array}{l}1,51 \mathrm{Aa} \\
1,53 \mathrm{Aa}\end{array}$ & $\begin{array}{l}30 \mathrm{~A} \\
27 \mathrm{~A}\end{array}$ & $\begin{array}{l}29 a \\
25 a\end{array}$ \\
\hline
\end{tabular}

(1) AD: preparo com arado de discos, CM: preparo com escarificador, SD: semeadura direta, AA: preparo com arado de aivecas, GD: preparo intensivo com grade. ${ }^{(2)}$ Letras mi núsculas na coluna comparam diferenças entre as duas profundidades de cada sistema de manejo e letras maiúsculas na linha comparam diferenças entre épocas dentro de cada profundidade nos diferentes sistemas de manejo, a $5 \%$ pelo testet. 
Quadro 3. Umidades correspondentes a $90 \%$ do limite de plasticidade (LP), a $-0,033 \mathrm{MPa}$, e a $90 \%$ de 0,01 MPa de um Latossolo Roxo, considerando o sistema de manejo, a profundidade e a época de amostragem

\begin{tabular}{|c|c|c|c|c|c|c|c|c|c|c|c|c|c|}
\hline \multirow{2}{*}{$\begin{array}{c}\text { Sistema } \\
\text { de manejo }\end{array}$} & \multirow{2}{*}{ Profundidade } & \multicolumn{4}{|c|}{ J aneiro/96 } & \multicolumn{4}{|c|}{ Abril/96 } & \multicolumn{4}{|c|}{ Novembro/96 } \\
\hline & & $\mathbf{L P}$ & $\begin{array}{c}90 \% \\
\text { do LP }\end{array}$ & $\begin{array}{l}-0,033 \\
\mathrm{MPa}\end{array}$ & $\begin{array}{c}90 \% \text { de } \\
-0,01 \mathrm{MPa}\end{array}$ & LP & $\begin{array}{r}90 \% \\
\text { do LP }\end{array}$ & $\begin{array}{l}-0,033 \\
\mathrm{MPa}\end{array}$ & $\begin{array}{c}90 \% \mathrm{de} \\
-0,01 \mathrm{MPa}\end{array}$ & LP & $\begin{array}{c}90 \% \\
\text { do LP }\end{array}$ & $\begin{array}{l}-0,033 \\
\mathrm{MPa}\end{array}$ & $\begin{array}{r}90 \% \text { de } \\
-0,01 \mathrm{MP}\end{array}$ \\
\hline & $\mathrm{m}$ & & & & & 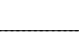 & - $\mathrm{k}$ & $\mathrm{kg}^{-1}$ & & & & & - \\
\hline$A D^{(1)}$ & $\begin{array}{r}0-0,07 \\
0,20-0,27\end{array}$ & $\begin{array}{l}0,32 \\
0,32\end{array}$ & $\begin{array}{l}0,29 \\
0,29\end{array}$ & $\begin{array}{l}0,28 \\
0,28\end{array}$ & $\begin{array}{l}0,30 \\
0,28\end{array}$ & $\begin{array}{l}0,34 \\
0,29\end{array}$ & $\begin{array}{l}0,31 \\
0,26\end{array}$ & $\begin{array}{l}0,28 \\
0,28\end{array}$ & $\begin{array}{l}0,28 \\
0,28\end{array}$ & $\begin{array}{l}0,33 \\
0,32\end{array}$ & $\begin{array}{l}0,30 \\
0,29\end{array}$ & $\begin{array}{l}0,29 \\
0,29\end{array}$ & $\begin{array}{l}0,30 \\
0,30\end{array}$ \\
\hline CM & $\begin{array}{r}0-0,07 \\
0,20-0,27\end{array}$ & $\begin{array}{l}0,34 \\
0,31\end{array}$ & $\begin{array}{l}0,31 \\
0,28\end{array}$ & $\begin{array}{l}0,29 \\
0,28\end{array}$ & $\begin{array}{l}0,30 \\
0,28\end{array}$ & $\begin{array}{l}0,34 \\
0,30\end{array}$ & $\begin{array}{l}0,31 \\
0,27\end{array}$ & $\begin{array}{l}0,28 \\
0,29\end{array}$ & $\begin{array}{l}0,30 \\
0,30\end{array}$ & $\begin{array}{l}0,33 \\
0,33\end{array}$ & $\begin{array}{l}0,30 \\
0,30\end{array}$ & $\begin{array}{l}0,29 \\
0,29\end{array}$ & $\begin{array}{l}0,31 \\
0,31\end{array}$ \\
\hline SD & $\begin{array}{r}0-0,07 \\
0,25-0,32\end{array}$ & $\begin{array}{l}0,32 \\
0,32\end{array}$ & $\begin{array}{l}0,29 \\
0,29\end{array}$ & $\begin{array}{l}0,28 \\
0,27\end{array}$ & $\begin{array}{l}0,28 \\
0,27\end{array}$ & $\begin{array}{l}0,33 \\
0,34\end{array}$ & $\begin{array}{l}0,30 \\
0,31\end{array}$ & $\begin{array}{l}0,27 \\
0,28\end{array}$ & $\begin{array}{l}0,28 \\
0,28\end{array}$ & $\begin{array}{l}0,34 \\
0,33\end{array}$ & $\begin{array}{l}0,31 \\
0,30\end{array}$ & $\begin{array}{l}0,29 \\
0,28\end{array}$ & $\begin{array}{l}0,30 \\
0,29\end{array}$ \\
\hline AA & $\begin{array}{r}0-0,07 \\
0,20-0,27\end{array}$ & $\begin{array}{l}0,33 \\
0,31\end{array}$ & $\begin{array}{l}0,30 \\
0,28\end{array}$ & $\begin{array}{l}0,28 \\
0,29\end{array}$ & $\begin{array}{l}0,28 \\
0,29\end{array}$ & $\begin{array}{l}0,32 \\
0,34\end{array}$ & $\begin{array}{l}0,29 \\
0,31\end{array}$ & $\begin{array}{l}0,28 \\
0,28\end{array}$ & $\begin{array}{l}0,30 \\
0,28\end{array}$ & $\begin{array}{l}0,31 \\
0,32\end{array}$ & $\begin{array}{l}0,28 \\
0,29\end{array}$ & $\begin{array}{l}0,29 \\
0,29\end{array}$ & $\begin{array}{l}0,30 \\
0,30\end{array}$ \\
\hline GD & $\begin{array}{r}0-0,07 \\
0,15-0,22\end{array}$ & $\begin{array}{l}0,34 \\
0,31\end{array}$ & $\begin{array}{l}0,31 \\
0,28\end{array}$ & $\begin{array}{l}0,27 \\
0,28\end{array}$ & $\begin{array}{l}0,29 \\
0,28\end{array}$ & $\begin{array}{l}0,30 \\
0,34\end{array}$ & $\begin{array}{l}0,27 \\
0,31\end{array}$ & $\begin{array}{l}0,28 \\
0,27\end{array}$ & $\begin{array}{l}0,28 \\
0,27\end{array}$ & $\begin{array}{l}0,32 \\
0,31\end{array}$ & $\begin{array}{l}0,29 \\
0,28\end{array}$ & $\begin{array}{l}0,28 \\
0,29\end{array}$ & $\begin{array}{l}0,30 \\
0,29\end{array}$ \\
\hline
\end{tabular}

(1) AD: preparo com arado de discos, CM: preparo com escarificador, SD: semeadura direta, AA: preparo com arado de aivecas, GD: preparo intensivo com grade.

executadas para umidades médias inferiores a $0,28 \mathrm{~kg} \mathrm{~kg}^{-1}$ (água retida a - 0,033 MPa) e 0,29 kg kg-1 ( $90 \%$ da água retida a - 0,01 MPa) para minimizar a compactação do solo. Em todos os casos, notou-se uma coerência entre os valores das umi dades ótimas de compactação obtidos pelo ensaio de Proctor normal e os obtidos pelos critérios da água retida a $-0,033 \mathrm{MPa}, 90 \%$ da água retida a - 0,01 M Pa (0,28 e $0,29 \mathrm{~kg} \mathrm{~kg}^{-1}$ ) e pelo critério de $90 \%$ do limite de plasticidade $\left(0,29 \mathrm{~kg} \mathrm{~kg}^{-1}\right)$.

\section{CONCLUSÕES}

1. Os sistemas demanejo estudados não afetaram significativamente a densidade do solo máxima e a umidade crítica de compactação.

2. Percebeu-se coerência entre os valores da umi dade crítica de compactação obti dos pelo ensaio de Proctor normal e os obtidos usando os critérios relativos a $90 \%$ do limite de plasticidade, $90 \%$ da água retida a -0,01 MPa, ea -0,033 $\mathrm{MPa}$.

3. Para o Latossolo Roxo estudado, as operações motomecanizadas devem ser executadas quando a umidade for menor do que $0,29 \mathrm{~kg} \mathrm{~kg}^{-1}$.

\section{LITERATURA CITADA}

CAMPBELL, D.J .; STAFFORD, J .V. \& BLACKWELL, P.S. The plastic limit, as determined by the drop-conetest, in relation to the mechanical behaviour of soil. J . Soil Sci., 31:11-24, 1980.
CAPUTO, H.P. Mecânica dos sol os e suas aplicações. 3.ed. Rio de J aneiro, Livros técnicos e científicos, 1973. v.1. 242p.

CASTRO NETO, P. Notas de aula práticas do curso de agrometeorologia. Lavras, Escola Superior de Agricultura de Lavras, 1982. 49p.

COMISSÃO DE FERTILIDADE DO SOLO DO ESTADO DE MINAS GERAIS - CFSEMG. Recomendações para uso de corretivos e fertilizantes do Estado de Minas Gerais; 4a aproximação. Lavras, 1989. 176p.

DAY, P.R. Particle fractionation and particle size analysis. In: BLACK, C.A., ed. Methods of soil analysis: physical and mineralogical properties, including statistics of measurements and sampling. Part. 1. Madison, Americam Society of Agronomy, 1965. p.545-567. (Agronomy, 9)

DIAS J UNIOR, M.S. Notas de aula de física do solo. Lavras, Universidade Federal de Lavras, 1996. 168p.

DIAS J UNIOR, M.S. \& PIERCE, F.J . O processo de compactação do solo e a sua modelagem. R. Bras. Ci. Solo, 20:175-182, 1996.

EKWUE, E.J . \& STONE, R.J . Organic matter effects on the strength properties of compacted agricultural soils. Trans. Am. Sci. Agric. Eng., 38:357-3365, 1995.

EKWUE, E.J . \& STONE, R.J . Density-moisturerelations of some Trinidadian soils incorporated with sewage sludge. Trans. Am. Sci. Agric. Eng., 40:317-323, 1997.

GAMERO, C.A. Efeito da mobilização do solo com enxada rotativa, sobre algumas de suas características físicas. Piracicaba, Escola Superior de Agricultura Luiz de Queiroz, 1982. 69p. (Tese de Mestrado) 
HOWARD, R.F.; SINGER, M.J . \& FRANTZ, G.A. Effects of soil properties, water content, and compactive effort on the compaction of selected California forest and range soils. Soil Sci. Soc. Am. J ., 45:231-236, 1981.

KLUTE, A. Water retention: laboratory methods. In: KLUTE, A., ed. Methods of soil analysis. 2.ed. Madison, American Society of Agronomy, 1986. v.1. p.635-662.

LATOUR, D.; LATOUR, K. \& WOLFINGER, R.D. Getting started with proc mixed, software sales and marketing. Cary, SAS institute Inc., 1994. 121p.

LARSON, W.E.; EYNARD, A.; HADAS, A. \& LIPIEC, J . Control and avoidance of soil compaction. In: SOANE, B.D. \& van OUWERKERK, C., eds. Soil compaction in crop production: developments in agricultura engineering. Amsterdan, Elsevier, 1994. p. 597-625p.

MANTOVANI, E.C. Compactação do solo. Inf. Agropec., 13:5255,1987

OHU, J .O.; RAGHAVAN, G.S.V.; MCHYES, E. \& MEHUYS, G. Shear strength prediction of compacted soils with varying organic matter contents. Trans. Am. Sci. Agric. Eng., 29:351355, 1986.

OJ ENIYI, S.O. \& DEXTER, A.R. Soil factors affecting the macrostructues produced by tillage. Trans. Am. Sci. Agric. Eng., 22:339-343, 1979.

PACHECO, A.A.R.C. \& DIAS J UNIOR, M.S. Estudo comparativo de métodos de campo e laboratório aplicados à confecção de blocos em adobe, Ci. Prát., 14:176-190, 1990.

PEREIRA, A.R. Efeitos da irrigação e mecanização sobre a compactação de um Latossolo cultivado com soja (Glycine max. L.). Lavras, Escola Superior deAgricultura de Lavras, 1994. 59p. (Tese de Mestrado)

RAGHAVAN, G.S.V.; MCKYES, E.; AMIR, I.; CHASSE, M. \& BROUGTON, R.S. Prediction of compaction due to off-road vehicletraffic. Trans. Am. Sci. Agric. Eng., 19:610-613, 1976.
RAIJ , B. van. \& QUAGGIO, J.A. Métodos de análise de solo para fins de fertilidade. Campinas, I nstituto Agronômico, 1983. (Bol etim Técnico, 81)

RESENDE, J .O. Compactação e adensamento do solo: metodologias para avaliação e práticas agrícolas recomendadas. In: CONGRESSO BRASILEIRO DE CIÊNCIA DE SOLO, 26., Rio de J aneiro, 1997. Palestras. Rio de J aneiro, SBCS/EMBRAPA, 1997. CD-ROOM.

ROCHA, G.C. Geologia, geomorfol ogia e pedol ogia de uma catena de solos situada no Campus da Escola Superior de Agricultura de Lavras, MG. Lavras, Escola Superior de Agricultura de Lavras, 1982. 109p. (Tese de Mestrado)

SEDIYAMA, G.C.; PTUITT, W.O.; COSTA, J.M.N. \& BERNADO, S. Modelo para computação de irrigação suplementar e do número de dias trabalháveis com máquinas agrícolas na produção da soja (Glycinemax L.). R. Ceres, 26:238-250, 1979.

SILVA, A.P.; LIBARDI, P.L.\& CAMARGO, O.A. Influência da compactação nas propriedades físicas de dois Latossolos. R. Bras. Ci. Solo, 10:91-95, 1986.

SOWERS, G.F. Consistency. In: BLACK, C.A., ed. Methods of soil analysis: Part 1. Physical and mineralogical properties, including statistics of measurements and sampling. Madison, Americam Society of Agronomy, 1965. p.391-399. (Agronomy, 9)

STANCATI, G.; NOGUEIRA, J.B. \& VILAR, O.M. Ensaios de laboratórios em mecânica dos solos. São Carlos, Universidade de São Paulo, Escola de Engenharia de São Carlos, 1981. 208p.

SAS. SAS/STAT. User's Guide. version 6. 4.ed. Cary, Sas I nstitute, 1990. v.2. 1686 p.

STONE, R.J . \& EKWUE, E.I. Maximum bulk density achieved during soil compaction as effected by the incorporation of three organic materials. Trans. Am. Sci. Agric. Eng., 36:17131719, 1993.

VARGAS, M. Introdução à mecânica dos solos. São Paulo, McGraw-Hill, 1977. 509p. 
L. H. A. FIGUEIREDO et al. 